\title{
SISTEMA DE GERENCIAMENTO DE DADOS COLETADOS PELA TECNOLOGIA RFID
}

\section{MANAGEMENT SYSTEM OF DATA COLLECTED BY THE RFID TECHNOLOGY}

\author{
Dieison Grumovski \\ Serviço Nacional de Aprendizagem Industrial (SENAI/SC) e AZUM Tecnologia Industrial, \\ E-mail: dieison.grumovski@sc.senai.br
}

Resumo: Este artigo tem como objetivo apresentar um sistema desenvolvido para gerenciar dados coletados através da tecnologia RFID (Radio Frequency Identification). Este sistema se faz necessário, devido a grande quantidade de dados que podem ser captados em intervalos de tempo muito pequenos, por vários modelos de equipamentos RFID. A tecnologia RFID esta revolucionando principalmente o segmento de logística, e o desenvolvimento deste sistema, permite a utilização de tecnologias/fabricantes distintos em um mesmo ambiente, beneficiando o desenvolvimento de projetos e a utilização desta tecnologia.

Palavras-chave: Middleware; RFID; Automação.

Abstract: The purpose of this article is to present a system developed to manage data collected through RFID technology. This system is necessary, because of the large amount of data that can be received in very short time intervals, by various models of RFID equipments. RFID technology is especially revolutionizing the segment of logistics, and the development of this system allows the use of technologies/manufacturers in the same environment, benefiting the development of projects and the use of this technology.

Key-words: Middleware; RFID; Automation. 


\section{INTRODUÇÃO}

Com o avanço da tecnologia busca-se encontrar soluções que tenham cada vez melhores resultados. Para isso, muitas vezes, existe a necessidade da integração entre diversos equipamentos e muitas vezes, de diversos fabricantes. Uma das tecnologias utilizadas para melhorar o desempenho de empresas é a Radio Frequency Identification (RFID). Esta tecnologia é aplicada em inúmeras áreas, principalmente para automação inteligente, podem-se destacar algumas áreas de aplicação, tais como, suply chain, pontes rolantes, esteiras e controle de patrimônio. (FAHL, 2005 e NOGUEIRA FILHO, 2005).

Com a utilização da tecnologia RFID nestas áreas, pode-se conseguir uma maior produtividade, vantagem competitiva, diminuir os erros humanos e uma maior segurança e visibilidade através do monitoramento dos produtos. $\mathrm{O}$ grande diferencial desta tecnologia é a leitura simultânea de diversos produtos e em velocidade superior a outras tecnologias com o mesmo fim, como por exemplo, o código de barras. (FAHL, 2005 e DRESCH JUNIOR, 2007).

Ao se trabalhar com equipamentos RFID de diversos fabricantes em um mesmo ambiente, surge um problema quanto a integração entre estes equipamentos. $O$ fato que faz com que isso aconteça é que praticamente todos os fabricantes de equipamentos RFID possuem um protocolo proprietário de comunicação entre os sistemas e o hardware.

Não existe nenhuma mobilização por parte dos fabricantes a fim de criar um protocolo padrão para comunicação entre os leitores e os sistemas e que possa ser seguido por todas as empresas. O que existe são empresas desenvolvendo sistemas de integração para os seus produtos em particular, como é o caso da empresa Creative Systems (2007), que desenvolveu uma solução RFID que utiliza um middleware para fazer a integração do hardware da empresa em com os aplicativos.

Uma solução interessante a ser desenvolvida consiste no desenvolvimento de um middleware capaz de integrar todos os equipamentos RFID de forma homogênea, independente das características do hardware ou do fabricante. Seu papel é fazer uma interface entre o hardware é as aplicações de forma que todos os equipamentos sejam vistos da mesma forma pelas aplicações, sem distinções entre fabricantes.

\section{TECNOLOGIA RFID}

O RFID é uma tecnologia que utiliza a rádio freqüência para a transmissão de dados. Esta tecnologia é composta basicamente por dois componentes, sendo eles: as etiquetas, também conhecidas como tags ou transponders e o sistema/dispositivo de leitura, 
que é composto pelo leitor e pela antena. As tags são responsáveis por armazenar e transmitir os dados para o dispositivo de leitura, que, por sua vez, tem a funcionalidade de energizar as tags, caso seja necessário, para que ela possa realizar a transmissão dos dados. Após os dados serem capturados pelo dispositivo de leitura, eles são enviados, geralmente, para um computador que possui um sistema capaz de tratar estes dados. (BHUPTANI; MORADPOUR, 2005 e GLOVER, 2007).

As tags podem ser divididas em três grupos: tags passivas, ativas e semi-passivas/semiativas. As tags ativas possuem uma bateria interna, o que permite a elas enviar os dados constantemente e serem lidas a maiores distâncias que as tags pertencentes aos demais grupos, não necessitando ser energizadas pelos dispositivos de leitura para poder enviar seus dados. As tags passivas não possuem bateria interna, por isso dependem da energia enviada pelo dispositivo de leitura para se auto-ativarem e enviarem seus dados. Este é um dos motivos pelo qual sua distância de leitura é menor comparado a das tags ativas. Outra grande diferença entre estes dois tipos de tags é quanto ao tempo de vida útil de cada uma, as tags ativas, por possuírem uma bateria interna, tem sua vida útil limitada, aproximadamente cinco anos, dependendo do ambiente em que ela é utilizada, como por exemplo, ambientes com grandes variações de temperatura podem interferir no seu tempo de vida útil. Já as tags passivas, considerando que não estão sujeitas à danos provocados por agentes externos, como por exemplo, batidas e exposição à água, sua vida útil seria indeterminada. As tags semi-passivas/semi-ativas são capazes de fornecer energia para o seu Circuito Integrado $(\mathrm{Cl})$, porém, não possuem energia suficiente para enviar os seus dados, dependendo assim, do envio de energia pelo dispositivo de leitura. O fato destas tags energizarem seus Cls, acelera o processo de envio dos dados em relação as tags passivas. (GLOVER, 2007 e DRESCH JUNIOR, 2007).

A grande maioria das tags já vem do fabricante com um código único gravado nelas, para que se possa fazer a identificação de forma exclusiva de cada item. Também, existem tags que permitem a gravação de dados, funcionando então como um banco de dados móvel, possibilitando a gravação de dados específicos de cada produto na própria tag, facilitando assim, a identificação do produto. (FAHL, 2005).

O leitor RFID é o responsável pela comunicação com as tags através de uma antena. Um leitor, geralmente, possui uma única antena, porém, existem leitores que podem conter mais de uma. Suas principais funções são energizar a tag, quando ela for passiva ou semipassiva, para que ela consiga enviar seus dados, retransmiti-los para um computador, e dependendo do leitor, ser capaz também de gravar dados nas tags, se elas permitirem. (BHUPTANI; MORADPOUR, 2005).

Como é o leitor RFID que energiza as tags, é importante analisar a frequência em que ele opera. A frequência define a taxa de transferência dos dados entre a tag e o leitor RFID, quanto maior a frequência, mais rápida a taxa de transferência. (BHUPTANI; 
MORADPOUR, 2005). As faixas de freqüência mais utilizadas pela tecnologia RFID são Low Frequency (LF ), High Frequency (HF) e Ultra High Frequency (UHF). (COUTO, 2003).

A divisão das faixas de freqüência pode ser observada no quadro 1.

\begin{tabular}{|c|c|c|c|c|c|c|}
\hline Frequências & $\mathrm{LF}$ & $\mathrm{HF}$ & VHF & UHF & SHF & EHF \\
$\begin{array}{c}\text { Faixas de } \\
\text { Frequência }\end{array}$ & $\begin{array}{c}30 \mathrm{KHz} \text { até } \\
300 \mathrm{KHz}\end{array}$ & $\begin{array}{c}3 \mathrm{MHz} \text { até } \\
30 \mathrm{MHz}\end{array}$ & $\begin{array}{c}30 \mathrm{MHz} \text { até } \\
300 \mathrm{MHz}\end{array}$ & $\begin{array}{c}300 \mathrm{MHz} \\
\text { até } 30 \mathrm{GHz}\end{array}$ & $\begin{array}{c}3 \mathrm{GHz} \text { até } \\
30 \mathrm{GHz}\end{array}$ & $\begin{array}{c}30 \mathrm{GHz} \text { até } \\
300 \mathrm{Ghz}\end{array}$ \\
\hline
\end{tabular}

Quadro 1: Faixas de frequência.

Fonte: Adaptado de (SYBASE, 2006)

A comunicação entre os leitores RFID e as tags, é realizada através das antenas presentes nos dois componentes. Dois dos fatores que determinam o alcance e a área de cobertura das antenas utilizadas são a potência e a forma de propagação do sinal emitido pela antena. Quanto maior é a potência maior é o alcance/área de cobertura. As formas de propagação mais utilizadas pela tecnologia RFID são circular e linear. A circular realiza uma leitura do ambiente mais abrangente, gerando um campo circular ao seu redor. Já a linear, a leitura é localizada, apenas em uma direção. (BHUPTANI; MORADPOUR, 2005).

Para exemplificar a diferença entre os equipamentos RFID, será realizado um comparativo entre quatro modelos de leitores RFID, que são também os modelos utilizados nos testes, são eles: LG120, LG120W, LG100, da empresa Azum tecnologia Industrial e o leitor Acuprox AP-08 da empresa Acura Technologies. (AZUM, 2008 e ACURA, 2008).

O leitor LG120 utiliza para comunicação a LF (125KHz), seu alcance de leitura é de até $10 \mathrm{~cm}$ e a forma de comunicação com outros equipamentos é via serial RS-232, porém, os leitores deste modelo possuem um conversor interno que permite a comunicação via ethernet, Transmission Control Protocol/Internet Protocol (TCP/IP). O formato das informações coletadas por este leitor tem como primeiro caracter, um caracter de controle, o código ASCII 2, depois vem o código da tag propriamente dito com 10 bytes, seguido de outro caracter de controle, o ASCII 3, ficando então com o seguinte formato: |ASCII 2| | 10 bytes do código da tag| |ASCII 3|.

O leitor LG120W utiliza freqüência LF $(125 \mathrm{KHz})$, seu alcance de leitura é de até $8 \mathrm{~cm}$ de raio e sua comunicação com o middleware é realizada via Wireless Fidelity (Wi-Fi). O formato dos códigos das tags coletadas por este leitor tem exatamente o mesmo formato dos códigos coletados pelos leitores do modelo LG120. 
O leitor LG100 utiliza para comunicação a UHF (433MHz), seu alcance de leitura é de até $4 \mathrm{~m}$ e a forma de comunicação com outros equipamentos é via serial RS-232. O formato das informações coletadas por este leitor é apresentado da seguinte forma: inicia-se com a string "ID=", seguido do código da tag propriamente dito com até 10 bytes, ficando então com o seguinte formato: $|I D=|$ | até 10 bytes do código da tag $\mid$.

Por sua vez o leitor Acuprox utiliza para comunicação a low frequency-LF (125KHz), seu alcance de leitura é de até 15 centímetros e sua comunicação com outros equipamentos é via serial RS-232. O formato das informações coletadas por este leitor é apresentado da seguinte forma: inicia-se com um caracter de controle ASCII 2, seguido de 10 bytes do código da tag, um caracter de controle "enter" ASCII 13 e do código ASCII 3, ficando então com o seguinte formato: |ASCII 2| |10 bytes do código da tag| |ASCI 13| |ASCII $3 \mid$.

No quadro 2 pode-se observar um comparativo das principais características dos leitores RFID apresentados.

\begin{tabular}{|c|c|c|c|c|}
\hline Leitores & Frequência & $\begin{array}{l}\text { Distância } \\
\text { de leitura }\end{array}$ & Comunicação & $\begin{array}{c}\text { Formato do código coletado } \\
\text { das tags }\end{array}$ \\
\hline LG120 & LF & Até $10 \mathrm{~cm}$ & $\mathrm{TCP} / \mathrm{IP}$ & $\begin{array}{c}\text { ASCII } 2<10 \text { bytes do código } \\
\text { da tag }>\text { ASCII } 3\end{array}$ \\
\hline LG120W & LF & Até 8 cm & Wi-Fi & $\begin{array}{c}\text { ASCII } 2<10 \text { bytes do código } \\
\text { da tag }>\text { ASCII } 3\end{array}$ \\
\hline LG100 & $\mathrm{HF}$ & Até $4 \mathrm{~m}$ & Serial RS-232 & $\begin{array}{c}\mathrm{ID}=<\text { até } 10 \text { bytes do código } \\
\text { da tag> }\end{array}$ \\
\hline Acuprox & LF & Até $15 \mathrm{~cm}$ & Serial RS-232 & $\begin{array}{c}\text { ASCII } 2<10 \text { bytes do código } \\
\text { da tag>ASCII } 13 \text { ASCII } 3\end{array}$ \\
\hline
\end{tabular}

Quadro 2: Comparativo entre leitores RFID.

Observando-se o quadro 2 é possível perceber as diferenças entre os leitores RFID, até mesmo entre os que pertencem ao mesmo fabricante. Estas diferenças, principalmente do formato das informações coletadas por estes dispositivos, dificulta a utilização desta tecnologia.

\subsection{Aplicações da tecnologia RFID}

As características da tecnologia RFID, permitem que ela seja aplicada em diversas soluções. Como por exemplo, controle de acesso, automação industrial e controle de estoque. 
Com a implantação de um sistema de controle de acesso, pode-se diminuir, por exemplo, a possibilidade de roubos, utilização de equipamentos por pessoas não autorizadas e o acesso de pessoas não autorizadas a locais restritos ou considerados perigosos. 0 controle de acesso utilizando a tecnologia RFID pode ser realizado através da utilização de tags embutidas em crachás e chaveiros, onde podem ficar armazenados além do código da tag, os dados da pessoa, bem como suas permissões de acesso. Nas portas das salas podem ser instalados leitores RFID para realizar a leitura dos crachás e/ou chaveiros e habilitar ou não o acesso dependendo das permissões de cada pessoa. (BHUPTANI; MORADPOUR, 2005 e PINHEIRO, 2006).

O uso da tecnologia RFID esta crescendo na área de automação industrial, principalmente no chão de fábrica, onde a maioria dos produtos passa por uma série e etapas desde o início até o final de sua produção. Pelo fato de cada etapa estar condicionada ao término de outra, com a utilização de dispositivos de leitura RFID em todas as etapas, é possível garantir, quando o produto chega a uma nova etapa, que ele passou pelas etapas anteriores. Pelo fato de existir um dispositivo de leitura em cada etapa, torna-se possível também localizar o produto, por exemplo, em uma linha de produção, verificando qual dispositivo realizou a última leitura do produto. Com a utilização do RFID na automação industrial, pode-se encaminhar os produtos para as etapas certas durante o processo de fabricação, diminuindo o tempo gasto para sua produção, bem como o manuseio destes produtos, podendo diminuir assim erros humanos, por exemplo. (BHUPTANI; MORADPOUR, 2005).

A tecnologia RFID empregada no controle de estoque possibilita um controle dos produtos que entram ou saem do estoque, que em conjunto com outras informações, como por exemplo, data de validade dos produtos, pode-se fazer um controle maior dos produtos, não permitindo, por exemplo, que os produtos passem da data de validade sem nem mesmo sair do estoque. A tecnologia RFID pode solucionar estes problemas, através de antenas posicionadas estrategicamente para realizar a cobertura de toda a área do estoque ou apenas das entradas e saídas do ambiente, portais logísticos, como mostra a figura 1. Com a utilização de antenas cobrindo toda a área do estoque, podem ser realizadas leituras constantes para atualização do estoque, estas leituras poderão ser realizadas diariamente e quantas vezes forem necessárias, sendo assim, produtos dificilmente serão esquecidos e perderão, por exemplo, sua data de validade. Com a utilização de leitores nos portais logísticos, pode-se saber a data na qual o produto entrou no estoque, quantos produtos estão no estoque, e a data da saída destes produtos do estoque, dados que também permitem gerar relatórios com informações sempre atualizadas. 


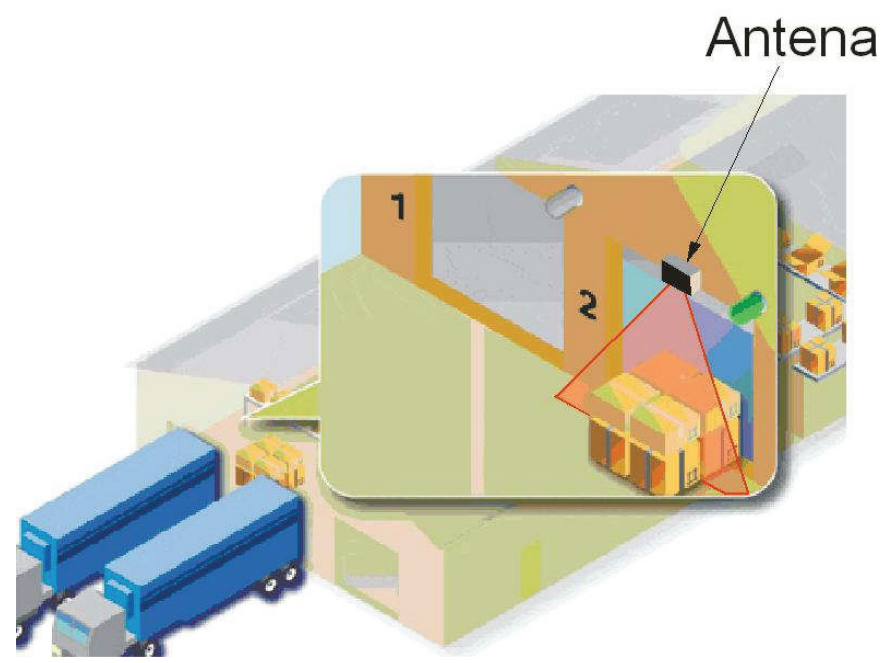

Figura 1. Leitura das tags contidas nos produtos passando por um portal logístico.

Fonte: (LIMA JUNIOR, 2006)

Apesar da tecnologia RFID ser facilmente aplicada em diversas soluções e trazer diversos benefícios, ela ainda possui uma série de restrições, como por exemplo:

a) o formato dos dados das tags que são coletados pelos dispositivos RFID, varia de acordo com cada equipamento e/ou fabricante;

b) os leitores RFID só permitem uma conexão por vez, ou seja, se um aplicativo estiver se comunicando com os dispositivos de leitura RFID, os outros aplicativos não podem se comunicar, somente um aplicativo por vez tem acesso aos dados coletados;

c) a forma de comunicação dos dispositivos de leitura com o computador onde os aplicativos são executados pode variar dependendo do leitor e/ou do fabricante do equipamento;

d) a tecnologia RFID pode gerar um grande fluxo de informações, este fluxo deve ser filtrado e gerenciado de modo que as aplicações só recebam os dados que realmente interessam.

Tendo em vista estas características da tecnologia RFID, conclui-se que é essencial a existência de uma inteligência capaz de gerenciar os dados coletados pelos sistemas de leitura. Esta inteligência é implementada na forma de uma camada de software, conhecida como middleware. 


\section{CONCEITOS BÁSICOS DE MIDDLEWARE}

O middleware é uma camada de software que é responsável por deixar a plataforma (sistema operacional e hardware) transparente para as aplicações. (BHUPTANI; MORADPOUR, 2005 e COULOURIS; KINDBERG; DOLLIMORE, 2005). Logo, com a utilização de um sistema com estas características, as aplicações não precisam se preocupar com a forma de comunicação entre os equipamentos RFID e o computador, nem com o formato das informações por eles disponibilizadas. Pode-se entender o conceito de middleware observando a figura 2, onde o middleware localiza-se entre os aplicativos e a plataforma, permitindo assim, que plataformas heterogêneas sejam transparentes para os aplicativos.

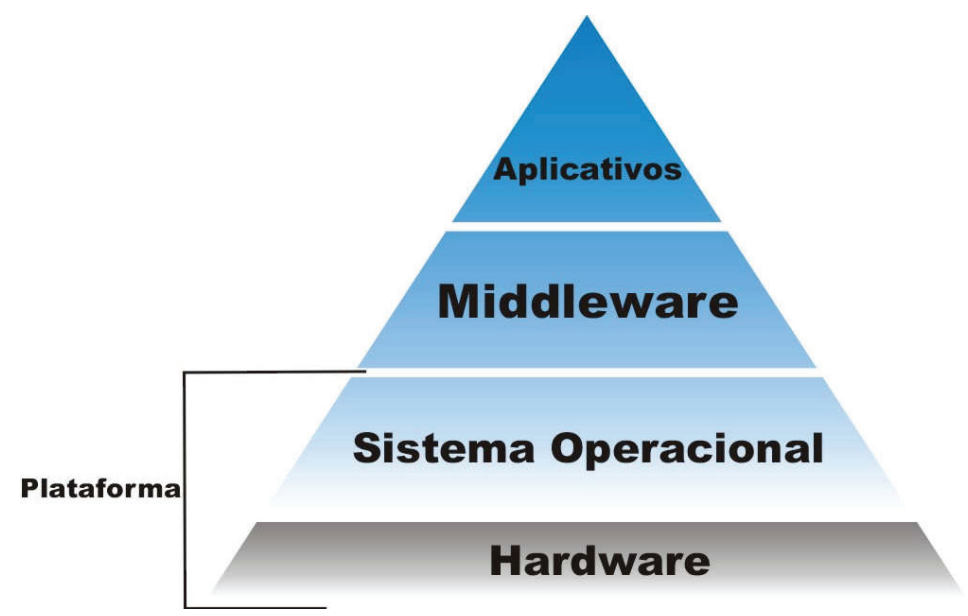

Figura 2: Posicionamento do middleware em relação à plataforma e aos aplicativos.

Segundo Bermstein (1996), um sistema middleware deve prover os seguintes serviços: gerenciamento de informações e de comunicação; atendimento das necessidades de um conjunto de aplicações de uma forma genérica e independente de plataforma; simplificação da comunicação de aplicativos através de abstrações e dar suporte para compartilhamento de recursos a aplicativos distribuídos.

Os middlewares podem ser baseados em modelos de arquitetura, estes modelos são apresentados a seguir.

\subsection{Middlewares Baseados em Modelos}

O desenvolvimento dos sistemas middlewares é geralmente baseado em algum modelo. Basicamente são quatro modelos: Transaction Processing Monitors (TPs); Remote Procedure Calls (RPCs); Message-Oriented Middlewares (MOMs); Object Request Brokers (ORBs). Cada um destes modelos é explicado na seqüência abaixo. 
Transaction Processing Monitors (TPs): os middleware baseados neste modelo tratam de aplicações de transações, executam computações como filtragens e verificações, e realizam atualizações em bancos de dados. Os TPs são utilizados, principalmente, no gerenciamento de dados e são a base de fornecimento de informação para os aplicativos. As atividades que ele realiza são basicamente coletar dados, processar estes dados, armazená-los, para que depois eles possam servir para geração de relatórios e documentos. (RODRIGUES, 2005).

Remote Procedure Calls (RPCs): permitem que clientes de uma aplicação possam executar procedimentos remotos como se fossem locais, daí o nome chamada de procedimentos remotos. Este modelo facilita a transparência e é adequado para aplicações clienteservidor. Porém este modelo depende do protocolo que esta sendo utilizado. Um grande problema deste modelo é que após o cliente enviar uma mensagem ao servidor, ele para e fica esperando uma resposta do mesmo, ou seja, trabalha de forma síncrona, isso causa uma perda de desempenho devido ao tempo de espera. (RODRIGUES, 2005).

Message-Oriented Middlewares (MOMs): esta arquitetura trabalha com troca de mensagens. Residem em ambas as partes de uma arquitetura cliente/servidor e permitem chamadas assíncronas (passos independentes) entre as aplicações. Os MOMs permitem que cada aplicação tenha uma fila exclusiva, onde são inseridas as suas mensagens. Caso uma aplicação de destino esteja indisponível, as mensagens são mantidas em uma fila correspondente a esta aplicação até que possa ser entregue. As mensagens podem conter um tempo de vida associado a elas, depois deste tempo, caso não sejam solicitadas, elas são descartadas. (RODRIGUES, 2005).

Object Request Brokers (ORBs): os ORBs permitem que objetos de aplicação sejam distribuídos em redes heterogêneas, ou seja, independem de linguagem de programação, de hardware ou software, de plataformas ou redes de comunicação. Este modelo fornece a abstração de que um objeto pode invocar métodos de outro objeto (objeto remoto), como se ambos estivessem em um mesmo computador. (COULOURIS; KINDBERG; DOLLIMORE 2005).

\subsection{Middlewares Desenvolvidos}

Um exemplo de middleware que está servindo como base para diversas implementações, é o middleware EPC, que pode ser conhecido também por Savant. As funcionalidades que compõe o middleware Electronic Product Code (EPC) são: coleta, processamento, filtragem e agregação dos dados. (BHUPTANI; MORADPOUR, 2005 e EPCGLOBAL, 2008). EPC é um número de série global, que identifica o produto de forma exclusiva. (EPCGLOBAL, 2008). Esta é a grande promessa do EPC, porém, existem diversos fabricantes que não seguem os padrões estabelecidos para o EPC, logo existe a necessidade de um middleware diferenciado para tratar destes casos. 
O middleware deve ser um sistema que interaja com os equipamentos RFID de forma "inteligente", pois certamente terá que sofre alterações para adaptar-se aos dispositivos, devido, por exemplo, às contínuas atualizações de firmware destes diferentes modelos de equipamentos provenientes de inúmeros fornecedores. O middleware deve ser flexível suficiente para se adaptar aos novos requisitos. Sabendo isso, o maior desafio é desenvolver uma camada de abstração que irá permitir que o middleware seja alterado caso novos dispositivos RFID sejam utilizados.

O middleware não necessariamente precisa ser baseado em um único modelo ou conter todas as características do modelo tido com base. Tendo em vista a necessidade da utilização de um middleware em conjunto com a tecnologia RFID, para suprir as limitações da tecnologia, foi desenvolvido um sistema de gerenciamento de coleta de dados baseado principalmente no modelo TP. Este sistema além de solucionar as restrições que a tecnologia RFID possui, ele oferece outras funcionalidade que permitem que as informações obtidas por esta tecnologia sejam melhores aproveitadas. No próximo item o sistema será explicado em detalhes.

\section{SISTEMA DE GERENCIAMENTO DE COLETA DE DADOS}

O sistema desenvolvido foi chamado de Sistema de Gerenciamento de Coleta de Dados (SGCD). O SGCD trabalha basicamente no gerenciamento das informações coletas pelos equipamentos RFID e na atualização de uma base de dados, que será consultada pelos aplicativos que desejarem ter acesso aos dados coletados. O SGCD também trabalha com o processamento de grandes quantidades de dados e realiza diversos procedimentos de forma repetitiva, como por exemplo, coleta dos dados e a verificação da validade destes dados, comparando os dados coletados por cada equipamento RFID com o seu formato padrão.

Devido a existência de diversas restrições da tecnologia RFID e como objetivo de aumentar a qualidade das informações que chegaram até aos aplicativos, foram desenvolvidas algumas funcionalidades para o SGCD, que são: transparência entre os aplicativos e a plataforma de hardware; gerenciamento do fluxo de dados coletados pelos dispositivos de leitura RFID; formatação das informações coletas; filtragens especifica de alguns códigos de tags coletados; adição de dados aos códigos das tags; gravação dos dados em uma base de dados.

Com a utilização do SGCD, caberá aos aplicativos apenas o trabalho de interpretar os dados de acordo com as suas funcionalidades. Se os dados provenientes dos dispositivos de leitura fossem enviados diretamente para os aplicativos, sem passar pelos filtros do SGCD, muito provavelmente os aplicativos não teriam capacidade de interpretá-los, 
pois uma grande quantidade de dados pode ser lida em um curto intervalo de tempo, e dependendo do fabricante das tags RFID, os códigos destas tags estarão em diferentes formatos.

Além do problema de interpretação dos dados coletados através da tecnologia RFID, sem a utilização do SGCD para se conectar aos dispositivos de leitura, eles só seriam acessados por um aplicativo de cada vez, pois eles não permitem mais de uma conexão por vez. Isto limitaria a utilização da tecnologia RFID. Através da figura 3 pode-se ver como ficou o acesso dos aplicativos aos dados coletados pelos dispositivos de leitura RFID.

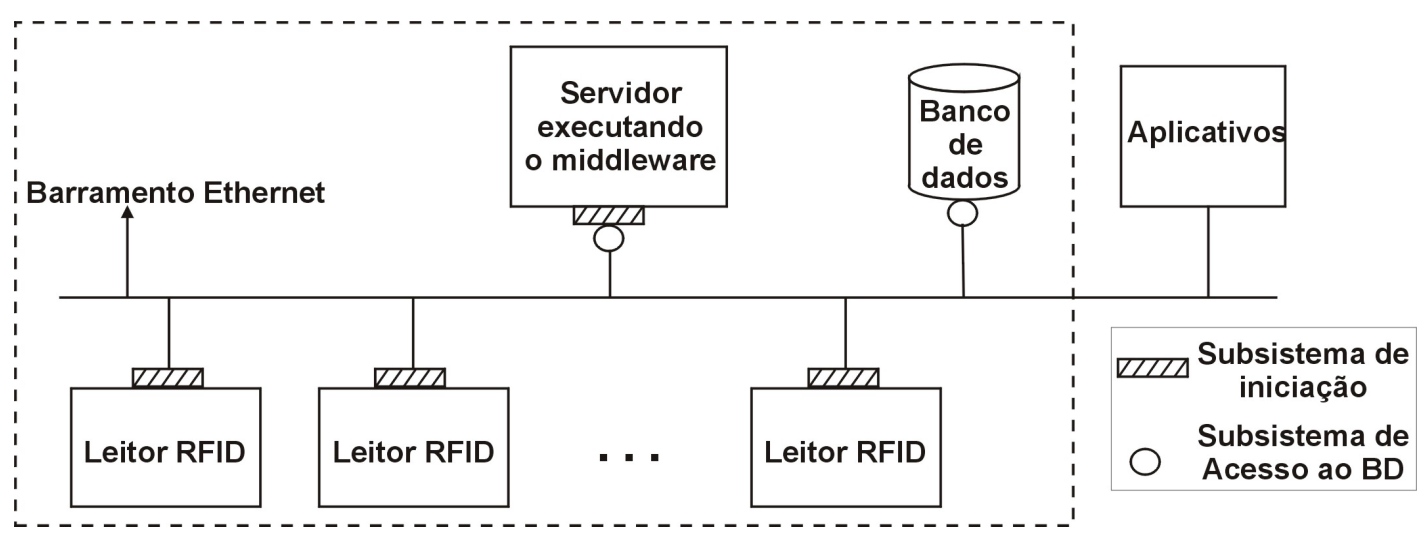

Figura 3. Aplicativos com acesso aos dados coletados pelos equipamentos RFID.

Através da figura 3 pode-se observar que todos os leitores RFID estão conectados a uma rede ethernet, os que não possuem comunicação ethernet, são conectados a um conversor que fará este trabalho. Desta forma todos os leitores RFID são acessados pelo SGCD, que por sua vez processa as informações e as disponibiliza através de um banco de dados para que os aplicativos tenham acesso as informações.

Com o objetivo de agregar ao sistema todas as funcionalidades mencionadas, o SGCD foi dividido em diversos módulos (subsistemas), onde cada módulo é responsável por uma tarefa do sistema. Tais módulos são os seguintes: de iniciação; de conexão; de coleta de dados; de filtragens especificas; para adicionar dados aos códigos das tags; de acesso ao banco de dados. 
Com base nos módulos desenvolvidos para o sistema, a figura 4 representa um diagrama de fluxo para o sistema.

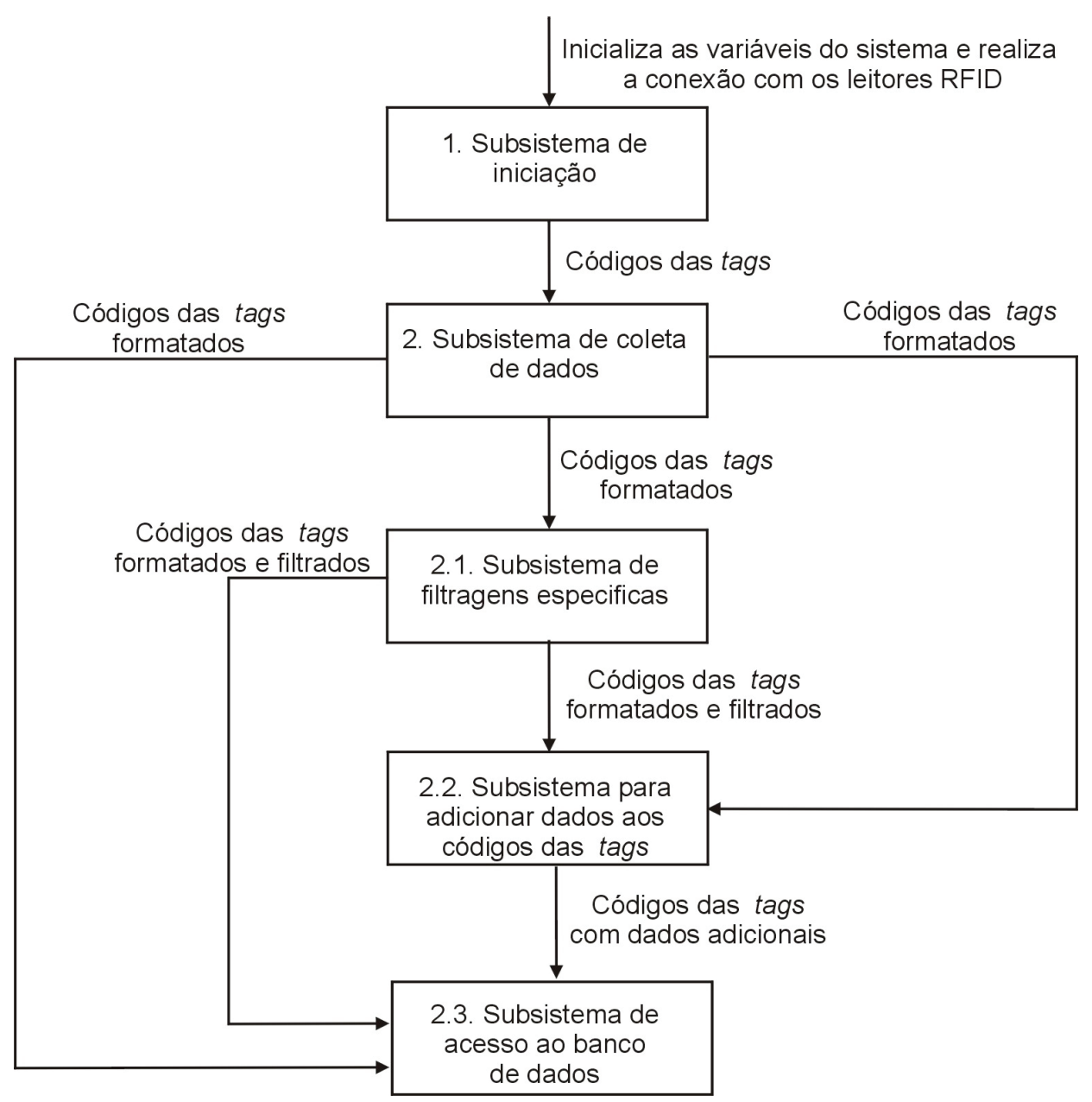

Figura 4: Diagrama de fluxo para o SGCD.

Todos os módulos, referenciados na figura 4 como subsistemas, serão explicados na seqüência.

\subsection{Módulo de Iniciação}

Na iniciação do $S G C D$, ele realiza a leitura de um arquivo de configuração no formato Extensible Markup Language (XML), que contém toda a configuração do sistema, desde a identificação dos dispositivos de leitura que estão conectados a ele até quais filtros serão executados. 
O XML foi escolhido por ser um padrão aberto, que tem como objetivo prover a flexibilidade para as aplicações. Com a utilização de um arquivo XML as buscas pelas informações se torna mais eficiente, não só pelo sistema, mas também pelos usuários, uma vez que terão que realizar toda a configuração do sistema através deste arquivo e o XML possibilita a nomeação das tags XML com qualquer nome, auxiliando assim no momento da atualização do arquivo ou na busca por informações.

O arquivo XML contém a especificação dos leitores RFID ao qual o SGCD se conecta, utilizando para tal, um nome designado para cada leitor (para identificálos individualmente) e o modelo do mesmo, bem como os dados necessários para estabelecer a conexão. Além destes dados, o arquivo XML, também é responsável por definir os filtros específicos que são executados pelo SGCD. Caso não exista necessidade de filtragens específicas, o conteúdo da tag XML < filtro> deve ficar vazio, com isso, tais filtros não são habilitados. $O$ arquivo $X M L$, também é responsável por informar ao SGCD as informações necessários para a conexão e inserção dos dados no banco de dados, como por exemplo, nome da base de dados e dados necessários para realizar o login nesta base.

Especificado desta maneira, o sistema é facilmente configurado através da atualização do arquivo XML. Nenhuma modificação em nível de linha de programação do sistema é necessária. Quando houver a necessidade da realização de alguma alteração de configuração de parâmetros do SGCD, esta alteração é realizada no arquivo XML, após ser realizada esta alteração é só reiniciar o sistema que ele realiza a leitura do novo arquivo XML automaticamente, já com as novas configurações.

Ao final da leitura do arquivo $\mathrm{XML}$, todas as variáveis responsáveis pela configuração do SGCD e pela conexão do mesmo com os dispositivos de leitura e com o banco de dados estão prontas (contendo seus valores de configuração). Com isto a conexão do SGCD com os dispositivos de leitura RFID pode ser iniciada.

\subsection{Módulo de Conexão}

Após o SGCD ter sido iniciado, e todas as variáveis do arquivo de configuração já terem sido carregadas para o sistema, o módulo de conexão, se encarregará de realizar a conexão do SGCD com os dispositivos de leitura RFID. Esta conexão é realizada através de uma comunicação via serial RS-232 ou via TCP/IP.

A transparência entre os dispositivos de leitura RFID e os aplicativos é uma das principais funcionalidades que viabilizam a utilização do sistema desenvolvido em conjunto com o sistema RFID, pois com a utilização do SGCD, as aplicações são desvinculadas da arquitetura física dos sistemas RFID, isso acontece pelo fato de ser o SGCD que se conecta ao hardware RFID e não os aplicativos. (GLOVER, 2007). 
O desenvolvimento deste módulo permite que vários aplicativos tenham acesso, ao mesmo tempo, aos dados coletados pela tecnologia RFID, tarefa que não é possível sem a utilização do SGCD, ou seja, o SGCD faz a multiplexação das informações.

Uma vez, o SGCD configurado e conectado aos dispositivos de leitura, é possível realizar a coleta dos códigos das tags. Esta tarefa é realizada pelo módulo de coleta de dados.

\subsection{Módulo de Coleta de Dados}

Este módulo do SGCD é responsável por coletar os códigos das tags, e também por formatá-los. Esta formatação, de certa forma, fará uso de filtros para realizar tal tarefa. A utilização de diferentes fabricantes e/ou equipamentos RFID em uma mesma aplicação, gera um problema quanto ao formato do código da tag, que pode variar de acordo com cada equipamento e fabricante. Por este motivo o SGCD implementa no momento da coleta dos dados, esta funcionalidade de formatação.

A tecnologia RFID é capaz de realizar leituras simultâneas de códigos de tags com alta velocidade e em curtos intervalos de tempo. Por estes motivos, é importante que o SGCD realize um controle do fluxo dos dados, que são coletados pelos dispositivos de leitura. À medida que os códigos das tags vão sendo recebidos pelo SGCD, eles vão sendo colocados em uma fila sem prioridade, de modo que nenhum código será perdido e que todos serão analisados pelos filtros obrigatórios. Tais filtros são responsáveis por verificar se o código da tag não esta corrompido, ou seja, verificar se é um código válido, e por retirar os caracteres adicionais (caracteres de controle), presentes nos códigos das tags, que, geralmente são caracteres utilizados para indicar o início e o fim do código propriamente dito. O formato considerado padrão, é o formato que não possui tais caracteres adicionais, ou seja, é somente o código da tag independente de quantos bytes contenha.

Após os dados terem sido filtrados por este módulo, eles estão formatados adequadamente e são válidos. Conforme a figura 4, os dados formatados podem ser coletados por três outros módulos, que são: o módulo de filtragens específicas, módulo para adicionar dados aos códigos das tags ou módulo de acesso ao banco de dados. Cada um deles é explicado na seqüência.

\subsection{Módulo de Filtragens Específicas}

Caso seja necessária, a realização de filtragens específicas dos códigos das tags para a aplicação na qual o SGCD está sendo empregado, os códigos formatados são enviados para o módulo de filtragens específicas. Os filtros específicos que o SGCD é capaz de executar são os seguintes: 
a) leituras duplicadas em um determinado período de tempo: Este filtro eliminará leituras duplicadas de um código de tag caso ele seja lido mais de uma vez em um determinado período de tempo (time out) pelo mesmo dispositivo de leitura. Por exemplo, se o time out, configurado no arquivo $\mathrm{XML}$, for igual a cinco segundos, após um código de tag ser lido por um dispositivo de leitura RFID, e armazenado no banco de dados pelo SGCD, este mesmo código de tag, lido pelo mesmo dispositivo de leitura, só poderá ser armazenado no banco de dados novamente, quando este dispositivo de leitura ficar pelo menos cinco segundos sem ler este mesmo código de tag;

b) eliminar uma das leituras, caso dois dispositivos de leitura leiam o mesmo código de tag, em um mesmo período de tempo: Este filtro é responsável por armazenar apenas um código de tag, caso este código, seja lido por dois dispositivos de leitura, em um determinado período de tempo. O código que será armazenado será o código que for lido por primeiro;

c) descartar leituras de códigos de tags que não pertencem a lista de códigos permitidos: Este filtro descarta os códigos de tags que foram lidos e que não pertencem a lista de códigos permitidos para a leitura. Esta lista pode ser gerada através de uma tabela no banco de dados e informada ao SGCD através do arquivo de configuração XML. Como exemplo de utilização do filtro, podese imaginar que em um ambiente existem tags cujos códigos variam de 1 até 10000, porém a aplicação a que o SGCD está sendo aplicado só necessita dos códigos de 1 até 5000, logo, se algum dispositivo de leitura ler um código de tag maior que 5000, o filtro irá descartar esta leitura (não irá armazená-la no banco de dados), diminuindo assim o volume de informações que serão processadas e armazenadas.

Após os dados serem filtrados pelo módulo de filtragens específicas, eles podem ser enviados para os módulos responsáveis por agregar dados aos códigos das tags, ou para o módulo de acesso ao banco de dados.

\subsection{Módulo para Adicionar Dados aos Códigos das Tags}

O módulo responsável por adicionar dados aos códigos das tags, pode receber os códigos das tags apenas formatados ou formatados e filtrados. Independente do tipo de dados que chegar até ele, sua funcionalidade é a mesma, adicionar dados aos códigos das tags. Os dados adicionais são: data e hora da leitura, e nome do leitor que realizou a leitura.

Para muitas aplicações não basta saber apenas o código da tag que foi lida, é importante também existir outras informações agregadas a este código, como por exemplo, para o 
controle de estoque é importante saber qual a data e hora em que o produto entrou no estoque, esta informação gera um controle maior da entrada e saída de mercadorias, e para uma linha de produção é importante saber qual foi o último leitor RFID que realizou a leitura, para saber se os produtos estão na etapa certa da produção e para facilitar a localização destes pela linha de produção. Por este motivo o SGCD é responsável por agregar tais informações adicionais aos códigos das tags, aumentando assim o número de aplicações aos quais os códigos das tags podem ser úteis.

Após realizada a adição de dados aos códigos das tags, tais dados são enviados para o módulo de acesso ao banco de dados.

\subsection{Módulo de Acesso ao Banco de Dados}

O módulo de acesso ao banco de dados é responsável por realizar a conexão do SGCD com o banco de dados, e armazenar os dados no mesmo. Estes dados podem chegar a este módulo e serem armazenados de quatro maneiras diferentes: códigos formatados; códigos formatados e filtrados; códigos formatados, filtrados e com dados adicionais; códigos formatados e com dados adicionais.

Os dados quando armazenados no banco de dados, são armazenados de forma seqüencial, ou seja, à medida que são lidos são armazenados, independente por exemplo do leitor que realizou a leitura ou da tags que foi lida.

O desenvolvimento do sistema em módulo é importante pois separa as funcionalidades do sistema, por exemplo, quando surgir a necessidade do SGCD se conectar a um novo modelo de dispositivo de leitura RFID e validar o código das tags que ele coletar, basta alterar o módulo de conexão, incluindo um novo drive de conexão para o novo hardware RFID e alterar o módulo de coleta de dados, que irá identificar e validar os códigos das tags. Todos os demais módulos continuam idênticos. Se um novo filtro especifico precisa ser criado, basta alterar o módulo de filtros específicos, os demais módulos permanecem sem alterações. Isso facilita quando for necessário fazer uma manutenção no sistema, ou uma atualização.

O principal desafio no desenvolvimento do SGCD foi identificar os leitores RFID, seu modelo, realizar a conexão com o mesmo e padronizar os códigos das tags. Com estas funcionalidades implementadas, o hardware ficou totalmente transparente para as aplicações.

A filtragem dos dados é bastante importante, pois, pelo fato da tecnologia RFID ser capaz de gerar grande quantidade de dados em intervalos de tempo muito pequenos, 
o processamento exigido do SGCD é consideravelmente alto. Com a utilização de filtros específicos, o fluxo de dados pode diminuir consideravelmente, e com isso diminuir o trabalho do SGCD.

A tecnologia RFID sendo utilizada sem a utilização de um sistema middleware, no caso o SGCD, fica consideravelmente limitada. Não existe a possibilidade de conexão de mais de um aplicativo ao mesmo hardware RFID, e nem validação das informações e controle do fluxo das mesmas que chegam até os aplicativos. Os testes foram realizados para comprovar os benefícios.

\section{VALIDAÇÃO}

Para comprovar os benefícios da utilização do SGCD em conjunto com a tecnologia RFID, foram realizados testes em laboratório com o auxilio de dois cenários distintos, portais logísticos e linha de produção/montagem, conforme figuras 5 e 6 respectivamente.

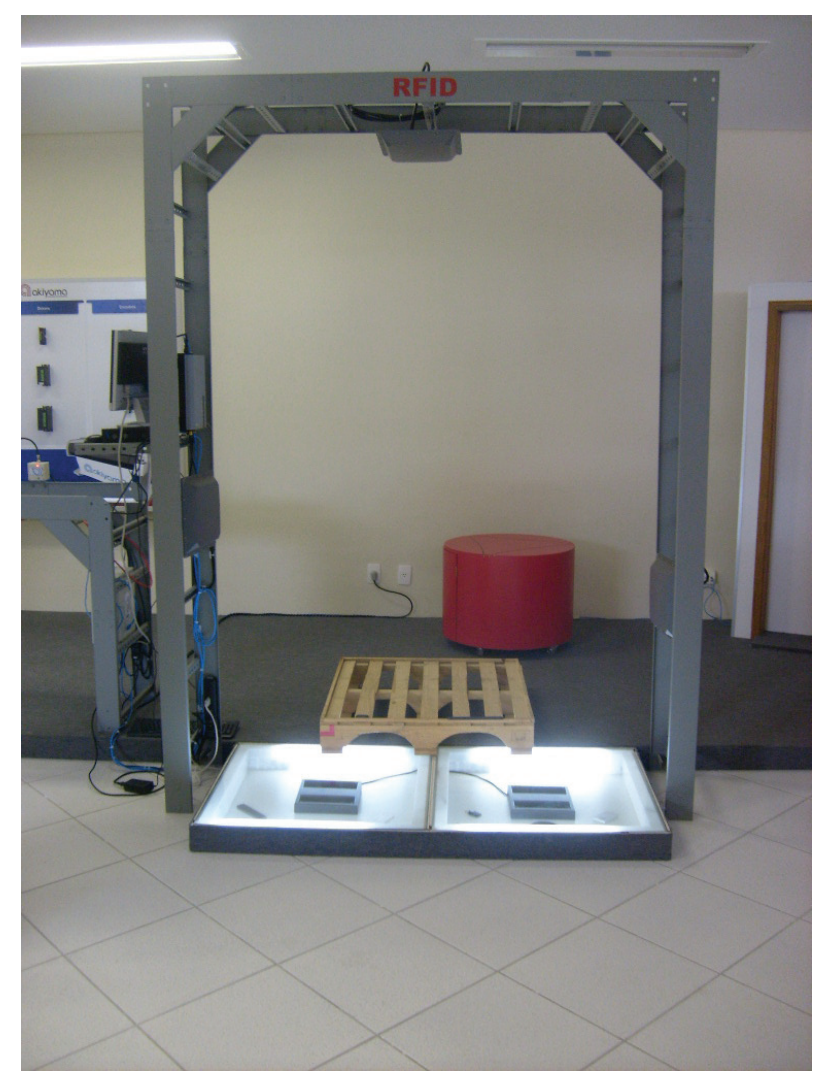

Figura 5. Portal logístico utilizado nos testes. 


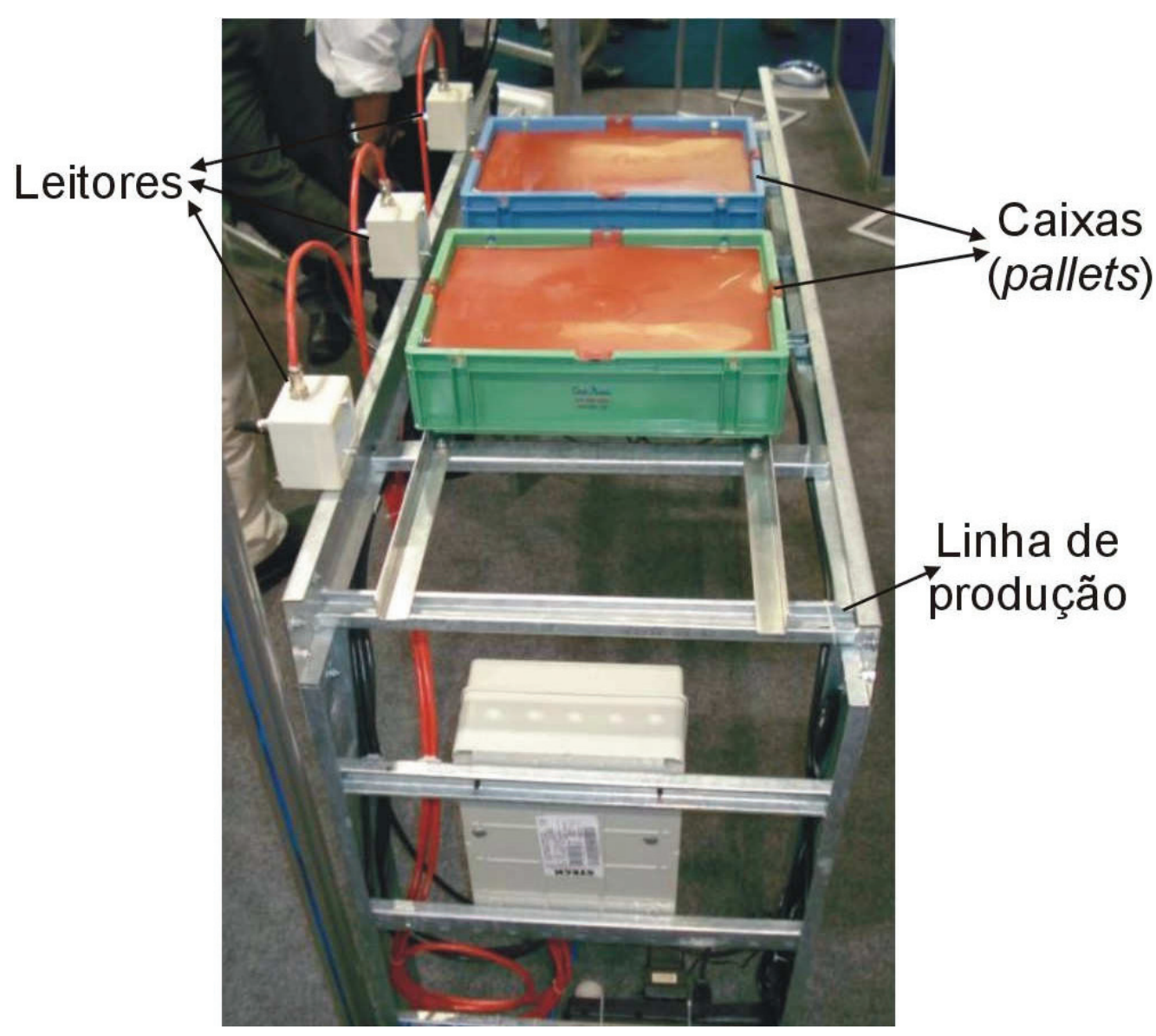

Figura 6. Linha de produção/montagem utilizada nos testes.

Em termos de características dos equipamentos, os cenários necessitam de equipamentos com freqüências distintas. Para o cenário de portais, é necessária a utilização de equipamentos RFID de alta freqüência, para assim cobrir uma área maior de leitura. Neste cenário não são necessários muitos leitores, dependendo do raio de leitura do leitor utilizado, apenas um já é o suficiente.

Para o ambiente de linha de produção os equipamentos devem ser de baixa freqüência, de modo que realizem as leituras em regiões mais restritas, pequenos raios de leitura, gerando assim áreas de leituras especificas, ou seja, a linha de produção ficará dividida em setores delimitados pelos próprios leitores RFID, facilitando a busca por produtos pela linha de produção, uma vez que bastará identificar qual leitor realizou a última leitura do produto para encontrá-lo.

O SGCD no ambiente de testes ficou posicionado de forma a receber os dados coletados pela tecnologia RFID instalada nos cenários de teste. Após a tecnologia RFID coletar os dados, ela os envia para o middleware, que irá processar e armazenar os dados em um banco de dados para que os aplicativos possam consultar o banco de dados e obter 
as informações coletadas pela tecnologia RFID, ou seja, apenas o middleware tem acesso direto a tecnologia RFID. Toda a informação proveniente desta tecnologia passa obrigatoriamente pelo middleware, é ele que realiza a conexão com os leitores RFID e controla toda a informação coletada, e as disponibiliza já de forma formatada, aos aplicativos através de um banco de dados.

Nos testes de conectividade, o SGCD se conectou aos quatro modelos de leitores disponíveis para os testes individualmente e simultaneamente sem apresentar nenhum problema. O recebimento e formatação dos códigos das tags lidas também foi realizado com êxito.

Para a realização dos testes no cenário de portal logístico, foi utilizado o leitor LG100 com tags ativas. Foram fixadas tags em caixas de papel simulando produtos, e foram realizadas cerca de 180 passagens com os produtos pelo portal, habilitando ou não os filtros específicos do middleware, em todas as passagens todas as tags que deveriam ser lidas foram identificadas e seus códigos foram armazenados no banco de dados de acordo com o formato especificado no arquivo XML de configuração.

No cenário de linha de produção foram utilizados os leitores LG120, Acuprox e LG120W. Os leitores foram fixados na linha de produção conforme já apresentado através da figura 6. Durante a realização dos testes foram simuladas diversas situações comuns em linhas de produção/montagem, são elas: mais de um leitor ler tags em um mesmo intervalo de tempo; produtos que não deveriam estar passando por aquela etapa da linha de produção/montagem, estavam passando. Para tratar este caso foi utilizado um dos filtros do middlewre, onde ele identifica as tags de acordo com um grupo de tags pré-cadastradas em uma tabela do banco de dados, caso algum produto necessite permanecer um determinado período de tempo em uma estação da linha de produção, neste intervalo de tempo, o leitor realiza diversas leituras da tag, porém, não existe a necessidade de processar e armazenar no banco de dados tantas informações, logo, para tratar este caso foi utilizado o filtro de time out desenvolvido para o middleware, onde após armazenar um determinado código de tag no banco de dados, ele só irá armazená-lo novamente caso o tempo configurado no filtro time out já tenha passado. Com isso diminuiu-se o tempo de processamento e o volume de dados armazenados no banco de dados.

Foram realizados testes com os leitores utilizados na linha de produção, para verificar a qual velocidade poderiam ser passados os produtos (as tags) pelo leitor, de modo que o mesmo conseguisse realizar a leitura dos códigos das tags e o middleware processar estes dados. 
Foram realizadas passagens de tags pelos leitores RFID, em diferentes velocidades. Pode-se verificar, observando a figura 7, que é necessário uma velocidade relativamente baixa para que o leitor consiga realizar as leituras em todas as passagens das tags. Na figura 7 pode ser observado que até uma determinada velocidade de leitura não ocorreram perdas de dados, ou seja, todas as tags foram lidas, porém, após a velocidade de $0,085 \mathrm{~m} / \mathrm{s}$, começaram a acontecer perdas, a medida que a velocidade aumentou, as perdas também aumentaram.

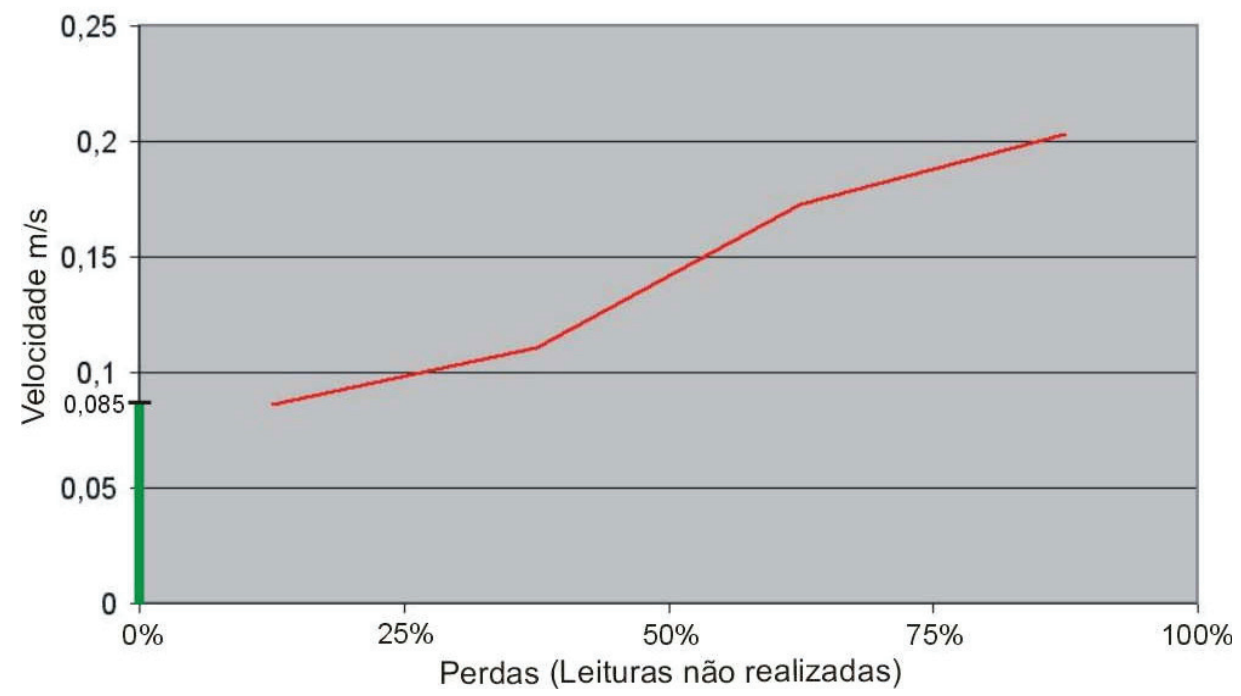

Figura 7: Velocidade da passagem das etiquetas pelo leitor RFID e suas perdas.

A realização deste teste foi importante pelo fato dele identificar os limites, quanto a velocidade de leitura, e que para esta tecnologia não basta apenas ter os equipamentos e realizar a instalação, é necessário fazer um estudo prévio que viabilize a sua utilização, ou seja, é importante que se identifique o ambiente e suas características antes da implantação do sistema para que falhas sejam evitadas e o sistema seja utilizado com máximo de acordo com os resultados esperados.

Foi desenvolvido também um pequeno aplicativo para comprovar a execução paralela do SGCD com diversas aplicações, onde todas as aplicações acessam simultaneamente os dados coletados pelos leitores RFID, sendo que isto não é possível sem a utilização do middleware.

Tal aplicativo foi executado duas vezes, ficando as duas aplicações sendo executadas simultaneamente com o middleware por várias horas. Todos os códigos de tags lidos pelos leitores RFID e enviados até o middleware, foram acessados pelos aplicativos. A realização deste teste comprova um dos principais benefícios que a utilização do middleware em conjunto com a tecnologia RFID oferece, a possibilidade de mais de uma aplicação ter acesso aos dados coletados pelos leitores RFID em um mesmo intervalo de tempo, contribuindo assim na viabilização de projetos utilizando esta tecnologia. 
Observando os resultados obtidos com a realização dos testes, pode-se concluir que o sistema middleware satisfaz as necessidades das aplicações quanto à necessidade de coletar os dados gerados pela tecnologia RFID, de forma simultânea e transparente.

Pode-se perceber que esta tecnologia é ideal para automatização de ambientes, principalmente industriais, como por exemplo, os ambientes trabalhados neste artigo, linhas de produção/montagem e portais logísticos. Esta tecnologia em conjunto com o middleware, aumenta a velocidade das linhas de produção, diminui erros provocados pela intervenção humana, facilita a localização dos produtos por toda a linha de produção/ montagem, elimina dados duplicados, através dos portais logísticos é possível saber quais produtos entraram e quais saíram dos armazéns, com isso é possível saber quanto tempo os produtos estão no estoque, evitando assim o vencimento destes produtos, e controlando a quantidade de produtos em estoque.

Todos estes benefícios juntos ajudam as empresas a alcançarem seus principais objetivos, diminuir gastos e aumentar os lucros.

\section{CONCLUSÕES}

O trabalho realizado e descrito neste artigo teve como objetivo especificar e desenvolver um sistema middleware capaz de gerenciar os dados coletados pela tecnologia RFID, de modo que esta tecnologia seja mais bem aproveitada.

Sem a utilização do middleware em conjunto com a tecnologia RFID, somente uma aplicação por vez pode se conectar e receber os dados de cada dispositivo de leitura, o que restringe o uso desta tecnologia. Diante das restrições da tecnologia RFID, o middleware foi desenvolvido visando prover: conectividade simultânea e transparente a modelos diferentes de leitores RFID; possibilidade da realização de filtragens dos dados coletados, diminuindo assim o volume dos mesmos; e por último, flexibilidade do middleware de modo que ele seja facilmente configurado.

A forma como o middleware é posicionado entre a tecnologia RFID e os aplicativos faz com que todos os sistemas de leitura sejam conectados ao middleware e que os dados coletados pelo RFID passem obrigatoriamente por ele, fazendo com que o middleware tenha o total controle de quais dados e em qual formato eles serão disponibilizados a todas as aplicações que os necessitam.

As maiores dificuldades no desenvolvimento deste trabalho foram: a aquisição de material para realização dos testes; a comunicação inicial do middleware com os leitores RFID; e a identificação e verificação dos dados enviados por esta tecnologia. 
Com o desenvolvimento deste trabalho, espera-se tornar a tecnologia RFID mais conhecida e estimular o seu estudo. Como sugestões de trabalhos futuros a este, podem ser citados:

a) desenvolvimento de um módulo para o middleware capaz de gerenciar os leitores RFID, realizando tarefas como, por exemplo, ligá-los, desligá-los e em casos do leitor possuir mais de uma antena o sistema deverá ser capaz de desabilitá-las ou habilitá-las. Com o desenvolvimento deste módulo, além de ser possível gerenciar os dados coletados pela tecnologia RFID, será possível gerenciar a própria tecnologia tornando o middleware uma ferramenta ainda mais completa;

b) desenvolvimento de um módulo para o middleware que seja capaz de estabelecer comunicação direta entre o middleware e as aplicações, de modo que o middleware possa controlar quais aplicações terão acesso aos dados e agilizar o recebimento das informações pelas aplicações.

\section{REFERÊNCIAS}

ACURA Technologies. Módulos RFID AP - 08. Disponível em: <http://www.acura.com. br/downloads/DS\%20Reader\%20AP-08.pdf>. Acesso em: 15 maio 2008.

AZUM Tecnologia Industrial. Disponível em: <http://azum.com.br>. Acesso em: 10 abr. 2008.

BERNSTEIN, P. A., Middleware: a model for distributed system services. Communications of the ACM, New York, vol. 39, no‥2. 1996, p. 86-98. Disponível em: <http://citeseer.ist.psu.edu/bernstein96middleware.html>. Acesso em: 01 ago. 2008.

BHUPTANI, M.; MORADPOUR, S. RFID: implementando o sistema de identificação por radiofreqüência. São Paulo, SP: IMAM, 2005. 250 p.

COULOURIS, G.; KINDBERG, T. ; DOLLIMORE, J. Distributed systems: concepts and design. 4th. ed. England: Addison-Wesley, 2005. 927 p.

COUTO, C. Identificação por radio freqüência. Material utilizado pelo departamento de eletrônica da universidade do Minho, Portugal, 2003. Disponível em: <http://www.dei. isep.ipp.pt/ qtdei/RFID_300403.pdf>. Acesso em: 11 abr. 2007. 
CREATIVE SYSTEMS. Throttleman com Solução RFID da Creativesystems. 2007. Disponível em: <http://www.creativesystems.pt/index.php?option=com_content\&task =view\&id=21\&ltemid=39>. Acesso em: 17 maio 2008.

DRESCH JUNIOR, A. Tecnologia RFID focando um sistema AIDC nas operações de armazenamento dos pallets. 2007. 59 f. Monografia (especialização) - Pontifícia Universidade Católica do Paraná, Curitiba, 2007.

EPCGLOBAL: organização responsável por supervisionar e desenvolver os padrões para a tecnologia RFID. Disponível em: < http://www.epcglobalinc.org/home>. Acesso em: 01 abr. 2008.

FAHL, C. R. Um estudo sobre a viabilidade de implantação de etiquetas inteligentes como vantagem competitiva em um centro de distribuição.2005. 95 f. Monografia (especialização) - Instituto Paulista de Ensino e Pesquisa, Campinas,SP, 2005.

GLOVER, B.; BHATT, H. Fundamentos de RFID. Rio de Janeiro, RJ: Alta Books, 2007. 227 p.

LIMA JUNIOR, L. F. A tecnologia de RFID no padrão EPC e soluções para implementação desta tecnologia em empilhadeiras. 2006. 90 f. Monografia (Especialização) - Pontifícia Universidade Católica de São Paulo, São Paulo, 2006.

NOGUEIRA FILHO, C. C. da C. Tecnologia RFID aplicada à Logística.2005.103 f.

Dissertação (Mestrado em Engenharia Industrial) - PUC-Rio, Rio de Janeiro, 2005.

PINHEIRO, J. M. dos S. Identificação por radio freqüência: Aplicações e vulnerabilidade da tecnologia RFID. Cadernos UniFOA, Volta Redonda, 2006. Disponível em: <http:// www.unifoa.edu.br/pesquisa/caderno/materias_ed2/18.html>. Acesso em: 01 abr. 2008.

RODRIGUES, Luís E. T. Tecnologias de Middleware. Instituto superior técnico-IST, Universidade técnica de Lisboa. 2005. Disponível em: http://homepages.di.fc. ul.pt/ ler/docencia/tm0405/. Acesso em:15 ago. 2008.

SYBASE. RFID Solutions Center: estado da Arte em RFID. [S.I.: s.n.], 2006. Disponível em:<http://www.sybase.pt/gvsview/gvs/sybasept/produtos_solucoes/docs/RFID_ Estado_de_Arte.pdf. 2006>. Acesso em: 11 maio 2007. 


\section{SOBRE O AUTOR}

Possui graduação em Ciência da Computação pela Universidade do Estado
de Santa Catarina, UDESC. Especialização em Engenharia de Produção pela
Faculdade Internacional de Curitiba, FACINTER - em andamento. Atua na
área de tecnologia e inovação da empresa TOTVS S/A e como professor
de informática no SENAI/SC em Joinville. Trabalhou como pesquisador da
tecnologia RFID (Identificação por Rádio Frequência) e como programador JAVA
no SENAl, unidade de Joinville. Trabalhou também como programador Java
nas empresas: AZUM Tecnologia Industrial, Quantus Tecnologia e IAControl
soluções empresariais.

